\title{
Effect of the Scalar Injection Mechanism on Passive Scalar Structure Functions in a Turbulent Flow
}

\author{
J. Lepore and L. Mydlarski* \\ Department of Mechanical Engineering, McGill University, 817 Sherbrooke Street West, Montréal, Québec, H3A 2K6, Canada
}

(Received 28 January 2009; published 17 July 2009)

\begin{abstract}
Higher-order passive scalar (temperature) structure functions are measured in the turbulent wake of a circular cylinder at a Taylor-microscale Reynolds number $\left(R_{\lambda}\right)$ of 370 . The scalar is injected by two different means: (i) heating of the cylinder and (ii) use of a mandoline. Even though the second-order statistics (e.g., power spectra, second-order structure functions) of the scalar field are experimentally indistinguishable in the inertial and dissipative ranges, we observe notable differences in the inertial-range scaling exponents $\left(\xi_{n}\right)$ of the scalar structure functions at higher orders. The implication is therefore that the variations in previous estimates of $\xi_{n}$ may be attributable to differences in the scalar field initial conditions (and may not be deemed characteristic of a universal nature of the small-scale statistics of turbulent passive scalars).
\end{abstract}

DOI: 10.1103/PhysRevLett.103.034501

Over the last $15-20$ years, there have been significant advances in the understanding of passive scalar mixing within turbulent flows. Some of these include (i) the realization that the internal intermittency (i.e., strong fluctuations in the dissipation rate) of a passive scalar arises independently of the velocity field, and (ii) the elucidation of the structure of passive scalar fields by studying multipoint correlation functions. In general, it has been concluded that many aspects of the scaling and structure of a turbulent passive scalar field arise from the mixing process itself, rather than from the nature of the velocity field that performs the mixing [1,2].

Of particular interest is the nature of the internal intermittency of a passive scalar field. As alluded to above, it is now known that passive scalars can exhibit internal intermittency when advected by Gaussian (i.e., nonintermittent) velocity fields [3-6]. Perhaps the most straightforward measures of internal intermittency of a passive scalar $(\theta)$ field are the structure function scaling exponents $\left(\xi_{n}\right)$. The latter are defined in terms of the structure functions:

$$
\left\langle\left(\Delta_{r} \theta\right)^{n}\right\rangle \equiv\left\langle[\theta(x)-\theta(x+r)]^{n}\right\rangle,
$$

where $r$ is the separation between two points and $n$ is the structure function order. In the inertial-convective subrange, it is observed that $\left\langle\left(\Delta_{r} \theta\right)^{n}\right\rangle \propto r^{\xi_{n}}$ for large enough Reynolds and Péclet numbers. Of particular interest is the dependence of the inertial-range structure function scaling exponent $\left(\xi_{n}\right)$ on the structure function order $(n)$. Kolmogorov-Obukhov-Corrsin (KOC) theory [7-9], which does not account for internal intermittency, predicts that $\xi_{n}=n / 3$. However, it is well known [2] that $\xi_{n}$ increases at a rate slower than $n / 3$ due to the effects of internal intermittency.

The objective of the present work is to determine the effect of the scalar injection method on the higher-order passive scalar structure function scaling exponents. KOC
PACS numbers: 47.27.Ak, 47.27.eb, 47.27.Jv, 47.27.wb

theory predicts that, in the limit of infinite Reynolds number, small-scale (i.e., inertial and dissipation range) statistics of the passive scalar field should be independent of the large-scale nature of the scalar field. Though KOC theory is extraordinarily successful in some respects (e.g., predicting the behavior of second-order quantities), it has other shortcomings, such as not accounting for the smallscale anisotropies of the scalar field in turbulent flows with mean scalar gradients [2]. The present work was motivated by that of Gylfason and Warhaft (2004) [10], who measured both longitudinal and transverse passive scalar structure functions (of orders 2 though 10) in grid turbulence with a mean scalar gradient over the Reynolds number range $150 \leq R_{\lambda} \leq 700$. They found no dependence of $\xi_{n}$ on the orientation of the separation $r$ (i.e., whether it be measured parallel or perpendicular to the direction of the mean scalar gradient), nor did they observe evidence for saturation of $\xi_{n}$ (i.e., $\xi_{n}$ tending to a maximum value) up to $n=10$. Lastly, in their compilation of data from previous research, they noted significant scatter in the values of $\xi_{n}$, which they attributed to either (i) convergence problems at higher orders, (ii) effects of flow or computational domain sizes causing clipping of large, rare fluctuations, or (iii) differences in initial or boundary conditions. The present work will answer whether the last of these factors may be responsible for the observed differences in $\xi_{n}$ determined by previous researchers. It will do so by measuring $\xi_{n}$ in two hydrodynamically identical flows, in which the passive scalar is injected using two different techniques.

The experiments described herein were conducted in the $85 \times 122 \times 274 \mathrm{~cm}^{3}$, low-background turbulence, opencircuit, suction-type wind tunnel in the Aerodynamics Laboratory at McGill University. The hydrodynamic wake was generated by placing a $7.30 \mathrm{~cm}$ diameter $(D)$ circular cylinder at the entrance of the test section. The 
scalar (temperature) field was generated by one of two methods: either by heating the cylinder or by use of a "mandoline" (introduced in [11]). In the former case, the cylinder was heated by a $1.5 \mathrm{~kW}$ heating element located at the cylinder's axis. In the latter case, an array of fine, parallel, heated wires (the mandoline) was placed downstream of, and oriented parallel to, the cylinder. Mandolines have also been used in [12-14] to study the passive scalar field in the turbulent wake of a cylinder. The mandoline used herein was constructed from thirteen 36 AWG (0.127 $\mathrm{mm}$ diameter) nichrome (type $A$ ) wires, spaced every $7.6 \mathrm{~mm}\left(=M_{\theta}\right)$, such that the mandoline width $(w)$ is $91.4 \mathrm{~mm}$. The wires were heated using a variable dc power supply providing a total power input of $1.2 \mathrm{~kW}$. The mandoline wires are small enough to have a negligible impact on the velocity field [12]. When in use, the mandoline was located at a downstream distance of $x_{\theta} / D=10$. A schematic of the experiment is given in Fig. 1.

Hot-wire anemometry and cold-wire thermometry were used to measure the longitudinal velocity and temperature fields, respectively. The longitudinal velocity component was measured using a DISA 56C01 constant-temperature hot-wire anemometer operated at an overheat of 1.8. The hot-wire sensor was made of $3 \mu \mathrm{m}$ diameter tungsten wire with a copper coating. The hot-wire calibration accounted for the variable temperature of the flow using a modified King's Law with temperature-dependent coefficients [15]. The temperature field was measured using a cold-wire thermometer built at Université Laval (Québec, Canada) based on a constant-current anemometer circuit [16]. The cold-wire sensor consisted of a Wollaston wire with a $0.63 \mu \mathrm{m}$ diameter platinum core operated with a current of $100 \mu \mathrm{A}$. Two types of measurements were made: (i) simultaneous velocity-temperature measurements consisting of $8.192 \times 10^{7}$ samples $\quad(=5000$ blocks $\times$ 16384 samples per block) of each quantity, and (ii) individual temperature measurements consisting of $1.2288 \times$ $10^{8}$ samples $(=7500$ blocks $\times 16384$ samples per block). All data were recorded at frequencies 2-2.5 times the lowpass filter frequency. For further details on the experimental method, see [12]. Finally, all measurements were recorded at a downstream distance of $x / D=30$, as well being directly downstream of the cylinder axis, and at the

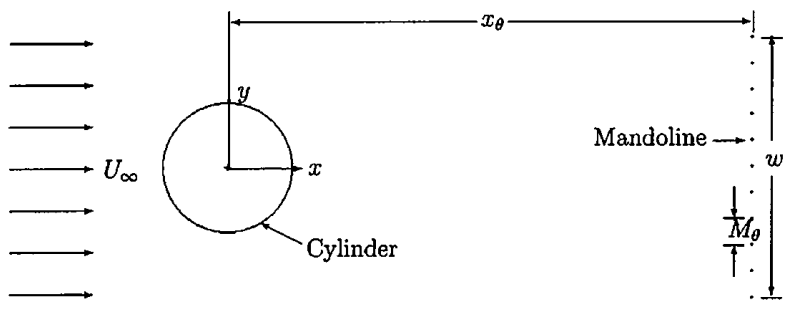

FIG. 1. Schematic of the experiment. The hot- and cold-wire sensors (not shown) were placed at $x / D=30$ and $y / D=0$. Reproduced from [12]. center of the wind tunnel $(y / D=0$ and $z / D=0$, respectively).

It is worth remarking that the measurement location is sufficiently far downstream for the small-scale details of the temperature's injection to be "forgotten." Probability density functions (PDFs) of the fluctuating temperature, $\theta$, (not shown) indicate that the scalar is well mixed at the current measurement location-i.e., the PDFs are smooth and unimodal in both cases. (If the scalar field were not well mixed, the PDF of $\theta$ would be bimodal, with a sharp peak corresponding to $T_{\infty}$ and another broader peak corresponding to temperature measurements in the poorly mixed thermal wake.) Furthermore, note that the mean temperature profiles (not shown) are smooth and quasiGaussian. (See [17] or [12], for example, for typical mean temperature profiles in thermal wakes generated by means of a heated cylinder or a mandoline, respectively.)

The flow parameters are summarized in Table I. Figure 2 shows the (nondimensional) power spectra of the velocity and temperature fields. Note the difference in the scalar field initial conditions, observed at low wave numbers in the power spectra of temperature. However, in the inertial and dissipative ranges, the temperature spectra are virtually indistinguishable, indicating the validity of KOC theory for second-order statistics. Furthermore, this observation validates the fact that the scalar field is well mixed at the measurement location. (Were this not the case, small-scale features relating to the two different scalar injection mechanisms would be observed.)

TABLE I. Flow parameters. The upper half of the Table describes the velocity statistics, whereas the lower half specifies the temperature statistics. Note that $U$ refers to the instantaneous longitudinal velocity, $u$ refers to the (instantaneous) longitudinal velocity fluctuation, and angular brackets denote time averages. $\ell$ and $\ell_{\theta}$ are the hydrodynamic and thermal integral length scales, respectively, $\varepsilon$ is the dissipation rate of turbulent kinetic energy, $\eta$ is the Kolmogorov length scale, and $\varepsilon_{\theta}$ is the smearing (or dissipation) rate of scalar variance. The cylinder diameter $(D)$ is $73.0 \mathrm{~mm}$, the kinematic viscosity $(\nu)$ is $15.7 \times$ $10^{-6} \mathrm{~m}^{2} \mathrm{~s}^{-1}$, and the thermal diffusivity $(\alpha)$ is $22.2 \times$ $10^{-6} \mathrm{~m}^{2} \mathrm{~s}^{-1}$.

\begin{tabular}{|c|c|c|}
\hline$U_{\infty}\left[\mathrm{m} \mathrm{s}^{-1}\right]$ & \multicolumn{2}{|c|}{10.3} \\
\hline $\operatorname{Re}_{D}\left\{=U_{\infty} D / \nu\right\}$ & \multicolumn{2}{|c|}{47700} \\
\hline$\langle U\rangle_{y / D=0}\left[\mathrm{~m} \mathrm{~s}^{-1}\right]$ & \multicolumn{2}{|c|}{8.42} \\
\hline$u_{\mathrm{rms}-y / D=0}\left[\mathrm{~m} \mathrm{~s}^{-1}\right]$ & \multicolumn{2}{|c|}{1.01} \\
\hline$\ell_{y / D=0}[\mathrm{~m}]$ & \multicolumn{2}{|c|}{0.132} \\
\hline$\varepsilon_{y / D=0}\left\{=15 \nu\left\langle(\partial u / \partial x)^{2}\right\rangle\right\}\left[\mathrm{m}^{2} \mathrm{~s}^{-3}\right]$ & \multicolumn{2}{|c|}{7.37} \\
\hline$\eta\left\{=\left(\nu^{3} / \varepsilon\right)^{1 / 4}\right\}[\mathrm{mm}]$ & \multicolumn{2}{|c|}{0.15} \\
\hline$R_{\lambda}\left\{=\left\langle u^{2}\right\rangle[15 / \nu \varepsilon]^{1 / 2}\right\}$ & \multicolumn{2}{|c|}{370} \\
\hline & Heat. Cyl. & Mand. \\
\hline$\theta_{\mathrm{rms}-y / D=0}[\mathrm{~K}]$ & 0.195 & 0.164 \\
\hline$\ell_{\theta-y / D=0}[\mathrm{~m}]$ & 0.134 & 0.052 \\
\hline$\varepsilon_{\theta y / D=0}\left\{=3 \alpha\left\langle(\partial \theta / \partial x)^{2}\right\rangle\right\}\left[\mathrm{K}^{2} \mathrm{~s}^{-1}\right]$ & 0.155 & 0.158 \\
\hline$\eta_{\theta}\left\{=\eta(\nu / \alpha)^{-3 / 4}\right\}[\mathrm{mm}]$ & \multicolumn{2}{|c|}{0.20} \\
\hline
\end{tabular}




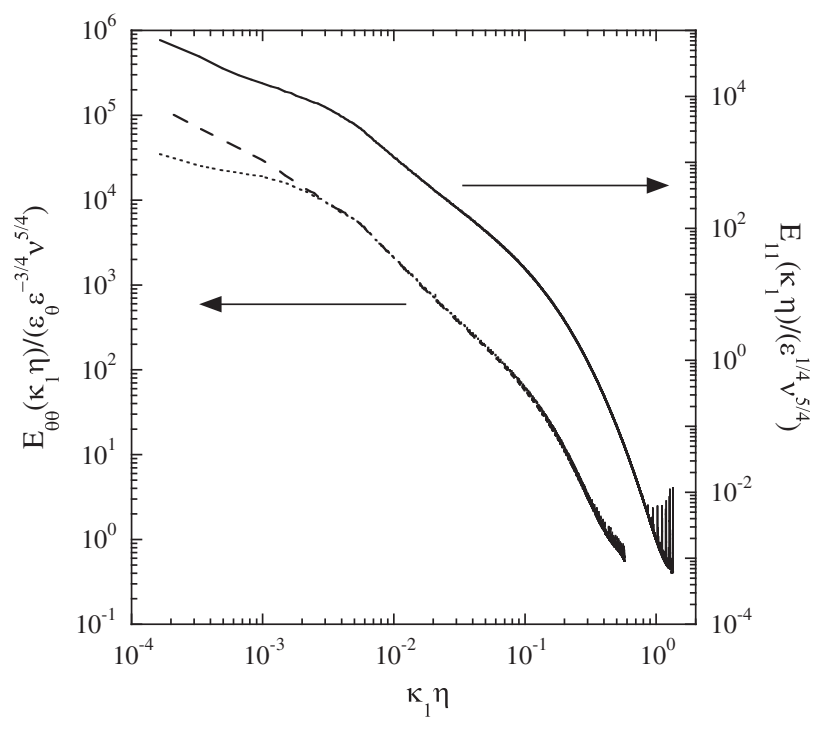

FIG. 2. One-dimensional longitudinal velocity (upper curve, right axis) and temperature spectra (lower curves, left axis), nondimensionalized by Kolmogorov variables. For the temperature spectra, the dashed line corresponds to the scalar field generated by the heated cylinder, whereas the dotted line corresponds to the case using the mandoline. $\kappa_{1}$ is the longitudinal wave number.

Figure 3 plots the measured (longitudinal) temperature structure functions for scalar fields generated using the heated cylinder and the mandoline. Note that they all exhibit clear inertial-convective subranges, approximately one decade in extent. Furthermore, note that the difference in initial conditions permeates increasingly farther down the turbulent cascade as the structure function order in-

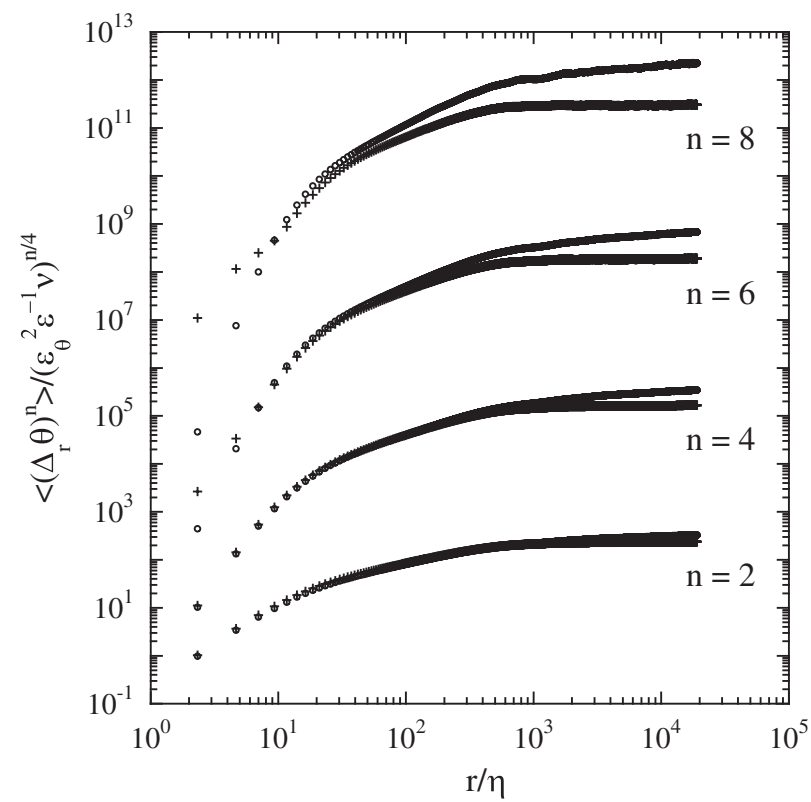

FIG. 3. Longitudinal temperature structure functions (of orders 2, 4, 6, and 8), nondimensionalized by Kolmogorov variables. $\bigcirc$ : Heated cylinder. +: Mandoline. creases. At second-order, the difference in the structure functions is effectively imperceptible in the inertial and dissipation ranges, whereas differences extend into the dissipation range at eighth order.

We furthermore verify the convergence of the data by considering PDFs of increments of $\theta$. Figure 4 plots $\left(\Delta_{r} \theta\right)^{n} P\left(\Delta_{r} \theta\right)$ for $n=8$. The area under the $\left(\Delta_{r} \theta\right)^{n} P\left(\Delta_{r} \theta\right)$ curve is equal to $\left\langle\left(\Delta_{r} \theta\right)^{n}\right\rangle$. It can therefore be observed from these curves that the integral is indeed closed at our highest order because we have recorded enough data to resolve the tails of the PDFs with sufficient accuracy.

Given our structure functions, the scaling exponents were calculated by least-squares fitting a power law to the inertial-convective subrange of the flow. The latter was determined from the mixed velocity-temperature structure functions (not shown), $\left\langle\left(\Delta_{r} u\right)\left(\Delta_{r} \theta\right)^{2}\right\rangle$, in analogy with the method of [18]. The compensated structure function $\left\langle\left(\Delta_{r} u\right)\left(\Delta_{r} \theta\right)^{2}\right\rangle /\left(-\frac{4}{3} \varepsilon_{\theta} r\right)$ was then plotted [19]. The two separations at which the compensated mixed structure function fell to $90 \%$ of its peak value were found and defined as the start and end of the inertial-convective subrange. Power laws were then fit to the structure function data between the two aforementioned separations.

The results are tabulated in Table II as well as plotted in Fig. 5. It is clear from both the table and the figure that the scaling exponents are notably different. Given that both flows are hydrodynamically identical, this can only be attributed to the different thermal injection mechanisms.

Lastly, Fig. 5 plots the present results along with those of other researchers. It is clear that there is considerable scatter in the results-an observation consistent with the hypothesis that $\xi_{n}$ depends on the scalar field initial or boundary conditions. Furthermore, note that the scatter in

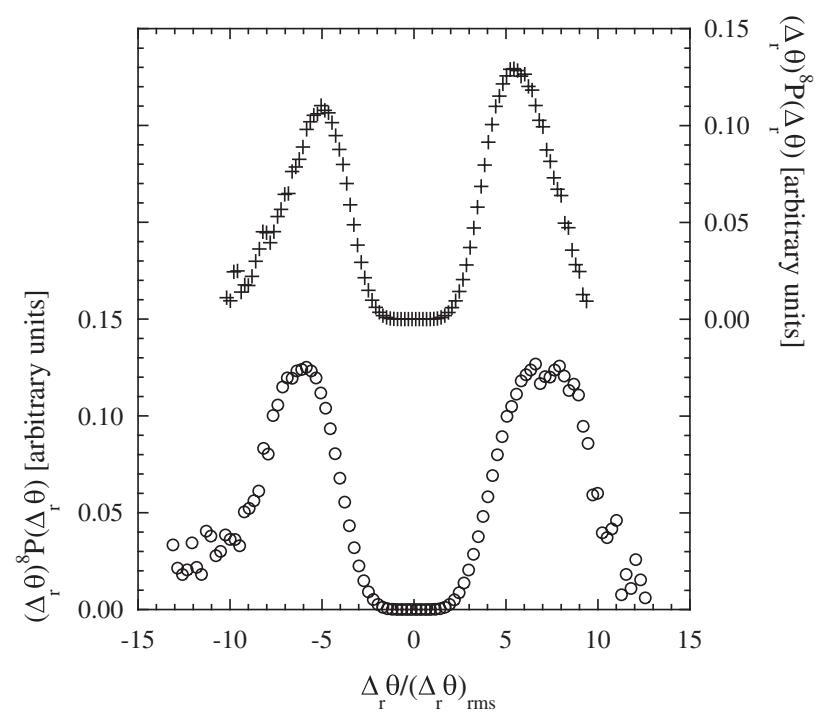

FIG. 4. $\left(\Delta_{r} \theta\right)^{8} \times \operatorname{PDF}\left(\Delta_{r} \theta\right)$ for an inertial-convective subrange separation of $r / \eta=65$. In the interest of clarity, the curves have been scaled. $\bigcirc$ : Heated cylinder (left axis). + Mandoline (right axis). 
TABLE II. Inertial-range velocity $\left(\zeta_{n}\right)$ and temperature $\left(\xi_{n}\right)$ structure function scaling exponents. The values listed are averages obtained from repetitions of the experiment. The error bars are defined as the maximum deviation between individual observations and their average.

\begin{tabular}{llll}
\hline \hline & $\zeta_{n}$ & \multicolumn{1}{c}{$\xi_{n}$} & \\
$n$ & & Heated Cylinder & Mandoline \\
\hline 2 & 0.74 & $0.63 \pm 0.005$ & $0.62 \pm 0.005$ \\
4 & 1.34 & $0.96 \pm 0.01$ & $0.94 \pm 0.02$ \\
6 & 1.83 & $1.19 \pm 0.01$ & $1.12 \pm 0.03$ \\
8 & 2.23 & $1.39 \pm 0.02$ & $1.23 \pm 0.04$ \\
\hline \hline
\end{tabular}

the compiled results for the scaling exponents of the velocity field, $\zeta_{n}$, is smaller.

Even though the second-order statistics of the scalar field are experimentally indistinguishable in the inertial and dissipative ranges, we observe notable differences in the inertial-convective range scaling exponents $\left(\xi_{n}\right)$ of the scalar structure functions at higher orders. This conclusion, though conflicting with KOC theory, is consistent with other contradictions, such as the persistent skewness of the scalar derivative (in the direction of a mean scalar gradient). Nevertheless, though the Reynolds number herein (as well as in most other experiments) is moderately high, there remains the possibility that the present results could derive from the finite Reynolds number of the flow. However, should this not be a low-Reynolds-number effect, the implication would be that variations in previous estimates of $\xi_{n}$ were attributable to differences in the scalar field initial conditions and therefore indicative of a lack of

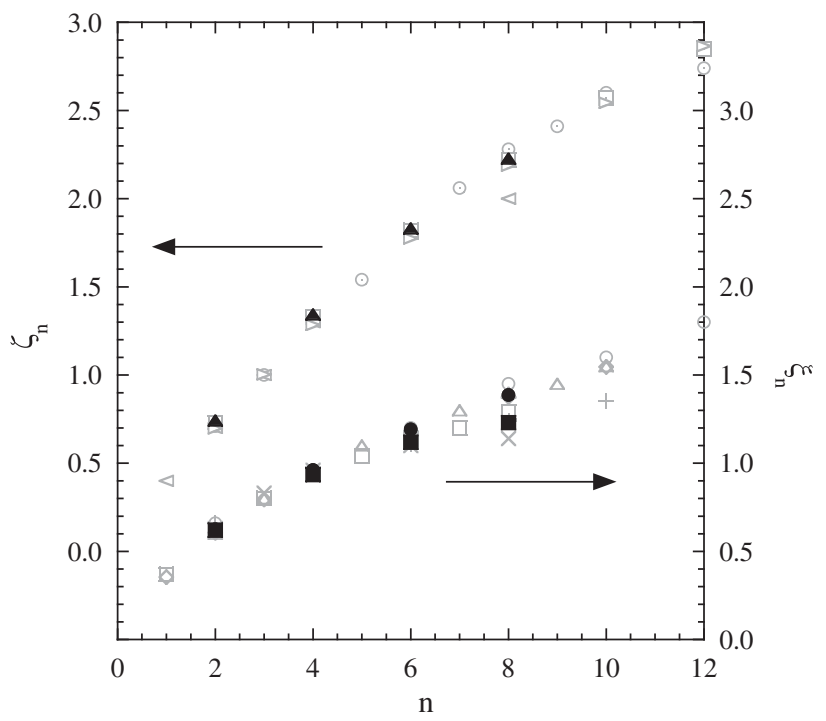

FIG. 5. Inertial-range scaling exponents for velocity, $\zeta_{n}$ (upper curves, left axis), and temperature, $\xi_{n}$ (lower curves, right axis).

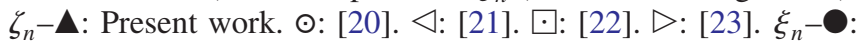
Present work, heated cylinder. $\mathbf{\square}$ : Present work, mandoline. $\bigcirc$ : [24]. $\times:$ [25]. $\square:$ [13]. +: [10]. $\diamond:$ [26]. $\triangle$ : [27]. small-scale universality for turbulent passive scalars. In either case, the present results demonstrate that previous estimates of $\xi_{n}$ (all measured or simulated at finite Reynolds numbers) are sensitive to the scalar field initial conditions and are therefore not representative of a universal behavior of turbulent passive scalar fields.

We thank Georges Tewfik and Mario Iacobaccio for their usual superior technical assistance. J. L. and L. M. gratefully acknowledge the Natural Sciences and Engineering Research Council of Canada for their generous, continued support.

*laurent.mydlarski@mcgill.ca

[1] B. I. Shraiman and E. D. Siggia, Nature (London) 405, 639 (2000).

[2] Z. Warhaft, Annu. Rev. Fluid Mech. 32, 203 (2000).

[3] M. Holzer and E. D. Siggia, Phys. Fluids 6, 1820 (1994).

[4] R. H. Kraichnan, Phys. Fluids 11, 945 (1968).

[5] R. H. Kraichnan, J. Fluid Mech. 64, 737 (1974).

[6] R. H. Kraichnan, Phys. Rev. Lett. 72, 1016 (1994).

[7] A. N. Kolmogorov, Dokl. Akad. Nauk SSSR 30, 301 (1941).

[8] A. M. Obukhov, Izv. Akad. Nauk. SSSR, Geogr. Geofiz. 13, 58 (1949).

[9] S. Corrsin, J. Appl. Phys. 22, 469 (1951).

[10] A. Gylfason and Z. Warhaft, Phys. Fluids 16, 4012 (2004).

[11] Z. Warhaft and J.L. Lumley, J. Fluid Mech. 88, 659 (1978).

[12] S. Beaulac and L. Mydlarski, Phys. Fluids 16, 3161 (2004).

[13] G. Ruiz-Chavarria, C. Baudet, and S. Ciliberto, Physica D (Amsterdam) 99, 369 (1996).

[14] E. Leveque, G. Ruiz-Chavarria, C. Baudet, and S. Ciliberto, Phys. Fluids 11, 1869 (1999).

[15] J.H. Lienhard, Ph.D. thesis, University of California at San Diego, 1988.

[16] J. Lemay and A. Benaissa, Exp. Fluids 31, 347 (2001).

[17] R. A. Antonia, Y. Zhou, and M. Matsumura, Exp. Therm. Fluid Sci. 6, 371 (1993).

[18] Jayesh, C. N. Tong, and Z. Warhaft, Phys. Fluids 6, 306 (1994).

[19] A. M. Yaglom, Dokl. Akad. Nauk SSSR 69, 743 (1949).

[20] F. Anselmet, Y. Gagne, E. Hopfinger, and R. Antonia, J. Fluid Mech. 140, 63 (1984).

[21] J. Maurer, P. Tabeling, and G. Zocchi, Europhys. Lett. 26, 31 (1994).

[22] A. Vincent and M. Meneguzzi, J. Fluid Mech. 225, 1 (1991).

[23] O. Boratav and R. Pelz, Phys. Fluids 9, 1400 (1997).

[24] R. A. Antonia, E. J. Hopfinger, Y. Gagne, and F. Anselmet, Phys. Rev. A 30, 2704 (1984).

[25] C. Meneveau, K. R. Sreenivasan, P. Kailasnath, and M. S. Fan, Phys. Rev. A 41, 894 (1990).

[26] S. Y. Chen and N.Z. Cao, Phys. Rev. Lett. 78, 3459 (1997).

[27] S. Y. Chen and R. H. Kraichnan, Phys. Fluids 10, 2867 (1998). 